Mon. Not. R. Astron. Soc. 000,18 (2002) Printed 8 November $2018 \quad$ (MN LATEX style file v2.2)

\title{
New bright optical spectrophotometric standards: A-type stars from the STIS Next Generation Spectral Library
}

\author{
C. Allende Prieto ${ }^{1,2 \star}$ and C. del Burgo ${ }^{3}$ \\ ${ }^{1}$ Instituto de Astrofísica de Canarias, 38205 La Laguna, Tenerife, Spain \\ ${ }^{2}$ Departamento de Astrofísica, Universidad de La Laguna, 38206 La Laguna, Tenerife, Spain \\ ${ }^{3}$ Instituto Nacional de Astrofísica, Óptica y Electrónica, Luis Enrique Erro 1, Sta. Ma. Tonantzintla, 72840 Puebla, Mexico
}

\begin{abstract}
Exoplanets have sparked interest in extremely high signal-to-noise ratio spectroscopic observations of very bright stars, in a regime where flux calibrators, in particular DA white dwarfs, are not available. We argue that A-type stars offer a useful alternative and reliable space-based spectrophotometry is now available for a number of bright ones in the range $3<V<8$ mag. By means of comparing observed spectrophotometry and model fluxes, we identify 18 new very-bright trustworthy A-type flux standards for the optical range (400-800 $\mathrm{nm}$ ), and provide scaled model fluxes for them. Our tests suggest that the absolute fluxes for these stars in the optical are reliable to within $3 \%$. We limit the spectral range to $400-800 \mathrm{~nm}$, since our models have difficulties to reproduce the observed fluxes in the near-infrared and, especially, in the near-UV, where the discrepancies rise up to $\sim 10 \%$. Based on our model fits, we derive angular diameters with an estimated accuracy of about $1 \%$.
\end{abstract}

Key words: stars: atmospheres; stars: fundamental parameters

\section{INTRODUCTION}

Absolute flux calibration of astronomical spectra has always proven challenging, in particular from the ground, but it is often crucial for understanding the nature of astronomical sources. Progress on this field has been summarized in the papers presented in two dedicated conferences (Sterken 2007; Milone \& Sterken 2011) and in a recent review by Bohlin et al. (2014).

Reliable standard stars are needed at all wavelengths. In the optical, efforts based on DA white dwarf modeling for HST calibration have paid off, and good consistency is found around the system based on three primary stars, and a longer list of secondary ones (Bohlin 2007, 2010, Bohlin \& Cohen 2008, Bohlin \& Koester 2008, and references therein). Those secondary stars span a range in Johnson $V$ approximately between -1 and 17 , but there are few calibrators available on both ends, at $V$ magnitudes brighter than 6 or fainter than 15. Cosmology surveys are now pushing the faint limit, and exoplanet research is focusing on stars significantly brighther than tenth magnitude.

In a previous paper, we contributed a number of faint DA white dwarf standards identified from the Sloan Digital Sky Survey (Allende Prieto, Hubeny \& Smith 2009). In this work we aim at covering the difficult area of the bright

* E-mail: callende@iac.es sources, at $V<8$ mag. No white dwarfs are known in this range, so other sources must be considered.

While any spectral type may be useful at very low resolution, standard calibrators usable over a wide range in spectral resolution, should have a smooth continuum. As for DA white dwarfs, being able to compute accurately the fluxes for a calibrator implies that its nature is well understood, discarding the presence of composite sources, significant interstellar extinction, or systematic observational errors. Atype stars seem the most natural kind of calibrators that are available to us in the range $0<V<10 \mathrm{mag}$.

A-type stars are warm enough that their spectra are relatively clean of metal lines in the optical range, although this is not true in the UV. A-type stars have fully radiative envelopes, avoiding the problem of convective energy transport, which is very hard to model accurately and can have an important influence on the thermal structure of deep photosphere where the continuum and the wings of Balmer lines form. With the shape of the continuum in the optical being dominated by photoionization of hydrogen from the $n=2$ level, modeling the spectral energy distribution of these stars in the visible is fairly independent of the detailed abundances of metals such as magnesium, silicon, or iron, which are important electron donors. Empirically, an excellent agreement has been found between observed and model spectral energy distributions of A-type stars in the 0.8 to $2.5 \mu \mathrm{m}$ range by Bohlin \& Cohen (2008).

On a negative note, A-type stars, unlike cooler dwarfs, 
tend to be young and rotate at a high rate. Fast rotation can drive the shape of the star away from a sphere, significantly altering the photospheric effective temperature and effective gravity across the surface of the star. For extreme cases, rotation can modify profoundly the spectral energy distribution or the photometric colors of a star (Pérez Hernández et al. 1999). Fortunately, as discussed by Gulliver, Hill \& Adelman 1994 for the case of Vega (see also Aufdenberg et al. 2006 and Hill, Gulliver \& Adelman 2010), despite there are spectral windows that are susceptible to rotation, the optical happens to be fairly insensitive to this parameter. It has also been found that a high fraction of A-type stars, in particular at young ages, have dusty circumstellar disks (e.g. Su et al. 2006, Currie, Plavchan \& Kenyon 2008), which may affect their energy distributions in the infrared but are innocuous in the optical. Very fast rotators can have gaseous disks, but these are not expected to alter the observed spectral energy distribution in the optical (Abt, Tan \& Zhou 1997). In summary, A-type stars can be suitable flux standards at visible wavelenghts, and are particularly useful to fill the gap left by DA white dwarfs at bright magnitudes.

The STIS Next Generation Spectral Library (NGSL; Gregg et al. 2006) includes spectrophotometry for 370 stars of a wide range of spectral types, including about 70 Atype stars with Johnson $V$ magnitudes spanning 2.9-10.3 mag. We have examined closely the A-type stars in this library and compared them with calculated fluxes based on Kurucz ODFNEW model atmospheres (Castelli \& Kurucz 2004). Taking good agreement between model fluxes and observations as indicative that the star is a normal A-type star and that the observations do not suffer from significant instrumental distortions, we have selected a number of stars that appear to be good candidates for flux standards. Similar to what has been done with DA white dwarfs (see, e.g., Bohlin 2007), model fluxes are scaled by reliable broad-band photometry to set the absolute scale, and if no photometric variability is found, the stars are promoted to spectrophotometric standards.

Section 2 describes the data and models used in this study. 33 is devoted to the analysis of observed spectral energy distributions (SEDs), and defines our criteria to select reliable standards based on the goodness-of-fit and the photometric stability of the sources. 4 focuses on the available photometry and the appropriate absolute scale for the stellar fluxes, while $\$ 5$ provides inferred angular diameters for our sample, which are compared to interferometric determinations available for a few objects. The paper closes with a short summary and our conclusions.

\section{OBSERVED AND MODEL SPECTRA}

The two main ingredients involved in our analysis are the STIS spectrophotometry from the NGSL, and synthetic spectra based on model atmospheres. We discuss below in 44 other observations used to check the absolute zero point to be adopted for the standard calibrators.

\subsection{Spectrophotometric observations}

Photometric and spectrophotometric observations from space benefit from a dramatic reduction in atmospheric ex- tinction and variability compared to data taken from the ground. In this spirit, an HST snapshot programme (GO 9088, 786, 10222) was granted observing time to obtain STIS spectrophotometry for 374 stars between $\sim 0.2$ and $1.0 \mu \mathrm{m}$ in cycles 10, 12, and 13 (Gregg et al. 2006). Several other successful GO and AR proposals aimed at improving the corrections for the slit throughput and other issues followed. The first version of the library was made public in 2008 and, with additional corrections, the second version was released in March 2010. Readers are referred to the online documentation provided with the library for details (Heap \& Lindler 2007; Lindler \& Heap 200\&1).

The data are fairly homogeneous, even though the wide spectral coverage required a combination of three different instrumental setups with three different gratings (G230LB, G430L and G750L). The expected resolving power $h^{2}$ varies slightly with wavelength, but is nominally about $500<R<$ 1100, as described in the STIS Instrument Handbook 3 .

The spectra were subject to a systematic analysis based on model spectra by Castelli \& Kurucz (2004) to derive atmospheric parameters, constrained by the parallaxes measured by the Hipparcos mission (van Leeuwen 2007) and stellar evolution models by Vandenberg et al. (2006). To some extent the analysis performed in $\$ 3$ duplicates this work, but we chose not to use parallaxes and stellar evolution models, given that we are mainly concerned with the models providing an accurate description of the stars' spectral energy distributions, and not so much unbiased atmospheric parameters. In fact, metallicity and surface gravity have a very limited impact on the spectral energy distribution of these stars in the wavelength range 400-800 nm. For example, the relative (normalized by their average value) fluxes of an A0V star like Vega change by less than half a percent when the surface gravity and metallicity vary by as much as 0.5 dex (del Burgo, Allende Prieto \& Peacocke 2010). Independent estimates of the atmospheric parameters for NGSL stars have been provided by Koleva \& Vazdekis (2012), with whom we compare results.

\subsection{Calculated spectral fluxes}

We have computed a grid of synthetic spectra covering the wavelength range $200-1000 \mathrm{~nm}$ with wavelength steps equivalent to $0.6 \mathrm{~km} \mathrm{~s}^{-1}$. The calculations are based on Kurucz ODFNEW model atmospheres (Castelli \& Kurucz 2004) and the synthesis code ASS $\epsilon$ T (Koesterke 2009; Koesterke, Allende Prieto \& Lambert 2008), operated in 1D (planeparallel geometry) mode. The reference solar abundances for the synthesis are from Asplund, Grevesse \& Sauval (2005), and these were scaled for elements heavier than helium according to the chosen metallicities. The micro-turbulence was set to $2 \mathrm{~km} \mathrm{~s}^{-1}$. While temperature and density are taken from the model atmospheres, the electron density is recalculated for consistency with the equation of state used, which includes the first 92 elements in the period table and

1 http://archive.stsci.edu/pub/hlsp/stisngsl/aaareadme.pdf

$2 R \equiv \lambda / \delta \lambda$, where $\delta \lambda$ corresponds to the FWHM of a Gaussian line spread function

3 http://www.stsci.edu/hst/stis/documents/

/handbooks/currentIHB/cover.html 
338 molecules (Tsuji 1964, 1973, 1976, with some updates). Partition functions are adopted from Irwin (1981).

Bound-free absorption from $\mathrm{H}, \mathrm{H}^{-}$, HeI, HeII, and the first two ionization stages of $\mathrm{C}, \mathrm{N}, \mathrm{O}, \mathrm{Na}, \mathrm{Mg}, \mathrm{Al}, \mathrm{Si}, \mathrm{Ca}$ (from the Opacity Project; see Cunto et al. 1993) and Fe (from the Iron Project; Bautista 1997; Nahar 1995) are included. Line absorption is included in detail from the atomic and molecular $\left(\mathrm{H}_{2}, \mathrm{CH}, \mathrm{C}_{2}, \mathrm{CN}, \mathrm{CO}, \mathrm{NH}, \mathrm{OH}, \mathrm{MgH}, \mathrm{SiH}\right.$, and $\mathrm{SiO}$ ) files compiled by Kuruct, but molecules are not important at the temperatures we are interested in. Level dissolution near the Balmer series limit is accounted for (Hubeny et al. 1994). The radiative transfer calculations include Rayleigh (H; Lee \& Kim 2004) and electron (Thomson) scattering. The damping of $\mathrm{H}$ lines is treated in detail using Stark (Stehlé 1994; Stehlé \& Hutcheon 1999) and self broadening (Barklem, Piskunov \& O'Mara 2000 for Balmer and Ali \& Griem 1966 for Lyman and Paschen lines). We used a grid with values of effective temperature in the range $7500 \leqslant T_{\text {eff }} \leqslant 13,000 \mathrm{~K}$, surface gravity $2.5 \leqslant \log g \leqslant 5.0$, and metallicity $-2.5 \leqslant[\mathrm{Fe} / \mathrm{H}] \leqslant+0.5$, with steps of $250 \mathrm{~K}$, 0.5 dex, and 0.5 dex, respectively. Spectra were convolved with a Gaussian kernel to account for instrumental broadening.

\section{SPECTRAL ANALYSIS}

We searched for the values of $T_{\text {eff }}, \log g,[\mathrm{Fe} / \mathrm{H}]$, and resolving power associated with the model fluxes that were closest to the NGSL spectra in the $200-1000 \mathrm{~nm}$ wavelength range for selected stars. A straight $\chi^{2}$ was used as merit function, making no difference between lines and continuum regions. Interstellar reddening was neglected, and we assumed that the correction of the Doppler velocities to the rest frame in the library is perfect, although we noticed slight inconsistencies in the wavelength scale of the spectra in the blue. The optimization was done using the FORTRAN90 code FERAE (Allende Prieto 2004, Allende Prieto et al. 2006, 2008, 2009), using the Nelder-Mead algorithm (Nelder \& Mead 1965). Model fluxes with any set of atmospheric parameters are derived by cubic Bézier interpolation (see Auer 2003 and included references) in the grid of model fluxes described in 2.2

\subsection{The prime A-type standard: Vega}

Vega offers an excellent opportunity to test our analysis procedure on a reference star that is actually considered an excellent standard. The Vega spectrum obtained with STIS and calibrated on the flux scale defined by three white dwarfs that constitute the set of primary HST spectrophotometric standards was found in excellent agreement with the fluxes predicted by Kurucz models with parameters $T_{\text {eff }} \simeq 9550$ $\mathrm{K}, \log g=3.95$, and $[\mathrm{Fe} / \mathrm{H}]=-0.5$ (Bohlin \& Gilliland 2004; see also García-Gil et al. 2005). More recently, after applying corrections for charge-transfer inefficiency in the STIS CCDs, the HST fluxes for this star have changed by up to $2 \%$, leading to a lower temperature estimate of about $T_{\text {eff }} \simeq 9400 \mathrm{~K}$ (Bohlin 2007).

4 kurucz.harvard.edu

5 Available from http://hebe.as.utexas.edu/ferre

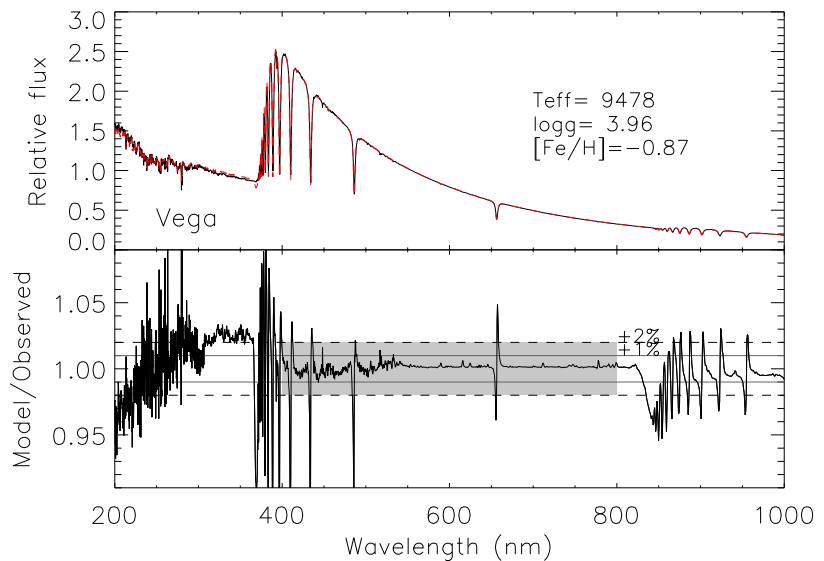

Figure 1. Upper panel: Observed (solid black line) and bestfitting model (broken red line) for Vega. Bottom panel: Ratio between model and observed fluxes.

We analyze the revised STIS spectrophotometry for Vega exactly in the same way as the NGSL A-type stars. We obtain $T_{\text {eff }}=9485 \pm 10 \mathrm{~K}, \log g=3.94 \pm 0.01,[\mathrm{Fe} / \mathrm{H}]=$ $-0.85 \pm 0.01$, and a resolving power $R=700 \pm 5$, with good agreement between model and observed fluxes, as ilustrated in Fig. 1, and a reduced chi-squared value of 3.6. These results are overall consistent with del Burgo et al. (2010). We adopted systematic and random error in the fluxes as provided by Bohlin \& Gilliland, where the former is $1 \%$ and dominates at the wavelengths used in our analysis. Our quoted uncertainties include random and systematic ones. Random uncertainties are derived either from the inversion of the curvature matrix, or from Monte Carlo experiments adding Gaussian noise to the spectrum, with very similar results, and we estimate systematic uncertainties by reanalyzing the spectrum after adding the maximum systematic error to the fluxes, with different sign at opposite extremes of our wavelength range, and intermediate values inbetween. Bohlin (2007) finds that the resolving power is $R \simeq 500$, slightly lower than our preferred value.

The average ratio $\langle F / f\rangle$ between the flux predicted by the model at the stellar surface and that observed in the 400-800 nm spectral window is then used to derive a stellar angular diameter of $\theta \simeq 2 R / d=2 \sqrt{f / F}=3.333 \pm 0.013$ mas. The random error contribution estimated from the scatter of the flux ratio across the selected wavelength interval is about 0.0004 mas, much smaller than the $0.8 \%$ uncertainty in the absolute magnitude in the $V$ band for Vega (Bohlin 2007), which sets the absolute flux calibration of the spectrum and dominates the error budget. This value is very similar to that inferred in the same fashion by Bohlin $\left(2007 ; \theta=3.335\right.$ mas if $T_{\text {eff }}=9400 \mathrm{~K}$ but $\theta=3.273$ mas if $T_{\text {eff }}=9550 \mathrm{~K}$ ), and to the results of Aufdenberg et al. (2006), $\theta=3.33 \pm 0.01$ mas using a model including distortions due to fast rotation. Monnier et al. et al. (2012) arrived at $\theta=3.324$ mas based on interferometric measurements, but see Peterson et al. (2006) for a discrepant result.

This excellent agreement gives us confidence that the analysis procedure and the assumptions involved are valid, but it should be noted that Vega is a rapidly rotating pole-on star and it may be somewhat variable - see the discussion of these and other problems with Vega by Gray (2007) and also 
Table 1. Derived parameters and angular diameters $(\theta)$ for the proposed bright spectrophotometric A-type standards. The scatter given corresponds to the standard deviation in $\theta$ for the spectral data points in the 400-800 nm range. The uncertainty $\sigma(\theta)$ is derived by adding the scatter in quadrature with a $0.4 \%$ error that corresponds to propagating the uncertainty in the zero-point of the flux scale $(0.8 \%$ or 0.008 magnitudes). Both the atmospheric parameters from the NGSL library (Lindler \& Heap 2008) and those derived in this paper are provided. We estimate our uncertainties are about $120 \mathrm{~K}, 0.2$ dex, and 0.1 dex (random), and $150 \mathrm{~K}, 0.2$ dex, and 0.1 dex (systematic), for $T_{\text {eff }}, \log g$, and $[\mathrm{Fe} / \mathrm{H}]$, respectively (but see text for a caveats about $\log g$ for some stars). The $V$-band photometry derived from the NGSL spectra, and those measured from the ground and compiled by Mermilliod et al. (1997) are in the last two columns.

\begin{tabular}{|c|c|c|c|c|c|c|c|c|c|c|c|}
\hline \multirow[b]{2}{*}{ Star } & \multirow[b]{2}{*}{$\begin{array}{r}\theta \\
\text { (mas) }\end{array}$} & \multirow[b]{2}{*}{$\begin{array}{r}\text { scatter } \\
(\text { mas })\end{array}$} & \multirow[b]{2}{*}{$\begin{array}{r}\sigma(\theta) \\
(\mathrm{mas})\end{array}$} & \multicolumn{3}{|c|}{ This work } & \multicolumn{3}{|c|}{ NGSL -} & \multirow[b]{2}{*}{$\mathrm{V}(\mathrm{NGSL})$} & \multirow[b]{2}{*}{$\mathrm{V}$ (phot.) } \\
\hline & & & & $\begin{array}{l}T_{\text {eff }} \\
(\mathrm{K})\end{array}$ & $\begin{array}{r}\log g \\
\left(\mathrm{~cm} \mathrm{~s}^{-2}\right)\end{array}$ & {$[\mathrm{Fe} / \mathrm{H}]$} & $\begin{array}{l}T_{\text {eff }} \\
(\mathrm{K})\end{array}$ & $\begin{array}{r}\log g \\
\left(\mathrm{~cm} \mathrm{~s}^{-2}\right)\end{array}$ & {$[\mathrm{Fe} / \mathrm{H}]$} & & \\
\hline HD 319 & 0.2786 & 0.0014 & 0.0018 & 8054 & 3.55 & -0.60 & 8195 & 3.86 & -0.39 & 5.9156 & $5.932 \pm 0.004$ \\
\hline HD 18769 & 0.2760 & 0.0020 & 0.0023 & 8057 & 3.63 & 0.15 & 8381 & 4.13 & 0.49 & 5.8980 & $5.905 \pm 0.014$ \\
\hline HD 34797 & 0.1242 & 0.0007 & 0.0009 & 12619 & 4.00 & -0.19 & 12884 & 4.06 & 0.02 & 6.5283 & $6.532 \pm 0.040$ \\
\hline HD 38237 & 0.1613 & 0.0010 & 0.0012 & 7901 & 3.60 & -0.07 & 8100 & 3.91 & 0.07 & 7.1495 & $\ldots$ \\
\hline HD 40573 & 0.1004 & 0.0007 & 0.0008 & 10065 & 4.21 & -0.30 & 10200 & 4.20 & -0.37 & 7.4679 & $\ldots$ \\
\hline HD 78316 & 0.2222 & 0.0011 & 0.0014 & 12747 & 3.79 & -0.26 & 12442 & 3.71 & -0.12 & 5.2410 & $5.233 \pm 0.008$ \\
\hline HD 79469 & 0.5027 & 0.0027 & 0.0034 & 10453 & 4.17 & -0.22 & 10489 & 4.23 & -0.18 & 3.8702 & $3.885 \pm 0.009$ \\
\hline HD 97633 & 0.7566 & 0.0036 & 0.0047 & 9136 & 3.50 & 0.01 & 9107 & 3.58 & -0.17 & 3.3031 & $3.338 \pm 0.023$ \\
\hline HD 110073 & 0.3078 & 0.0024 & 0.0027 & 12086 & 3.81 & -0.78 & 12041 & 3.77 & -0.37 & 4.6605 & $4.638 \pm 0.004$ \\
\hline HD 141795 & 0.7559 & 0.0057 & 0.0065 & 8094 & 3.76 & 0.07 & 8418 & 4.21 & 0.34 & 3.6976 & $3.708 \pm 0.009$ \\
\hline HD 141851 & 0.3975 & 0.0020 & 0.0026 & 8135 & 3.77 & -0.31 & 8231 & 3.96 & -0.22 & 5.1075 & $5.105 \pm 0.005$ \\
\hline HD 164967 & 0.1529 & 0.0012 & 0.0013 & 8153 & 3.84 & -0.75 & 8533 & 4.07 & -0.60 & 7.1957 & $\ldots$ \\
\hline HD 166991 & 0.1730 & 0.0008 & 0.0011 & 8347 & 3.86 & -0.29 & 8496 & 4.00 & -0.26 & 6.8107 & \\
\hline HD 174240 & 0.2061 & 0.0013 & 0.0016 & 8894 & 3.69 & -0.26 & 9274 & 3.76 & -0.18 & 6.2177 & $6.240 \pm \ldots$ \\
\hline HD 196426 & 0.1484 & 0.0007 & 0.0009 & 12376 & 3.85 & -0.63 & 12800 & 3.95 & -0.48 & 6.2023 & $6.211 \pm 0.015$ \\
\hline HD 201377 & 0.2020 & 0.0012 & 0.0014 & 7905 & 3.59 & -0.10 & 8084 & 3.92 & 0.04 & 6.6725 & \\
\hline HD 204041 & 0.2254 & 0.0011 & 0.0014 & 7999 & 3.86 & -0.90 & 8259 & 4.17 & -0.60 & 6.4354 & $6.455 \pm 0.005$ \\
\hline HD 205811 & 0.1977 & 0.0009 & 0.0012 & 9294 & 4.12 & -0.00 & 9286 & 4.20 & -0.13 & 6.1654 & $6.193 \pm 0.011$ \\
\hline
\end{tabular}

Bohlin (2014) - limiting the extend to which the simple hydrostatic plane-parallel models used here can reproduce its spectral energy distribution. Note that the predicted fluxes are systematically higher in the region between 280 and 370 $\mathrm{nm}$, in line with the expected corrections for rotationally distorted models (Gulliver, Hill \& Adelman 1994).

\subsection{The bright A-type stars in NGSL}

The analysis of the NGSL A-type stars was carried out exactly in the same way as Vega's. We obtained parameters for all of them and, in general, acceptable fittings to the observed spectral energy distributions. The analysis was performed both on the first (v1) and the second (v2) versions of the NGSL. The differences in the quality of the fittings were modest, so we decided to adopt the results based on the updated version (v2).

We find values for the resolving power of the spectra in the library between 460 and 730 , but with a narrow distribution, centered at $R=534$, with a standard deviation of 60 , and therefore we decided to force $R=500$ in the final fittings. We compared the results for the parameters determined with the individual $R$ values for each star and $R=500$ and found negligible differences. We also examined spectra with higher resolution from the MILES library (Falcón-Barroso et al. 2011 and references therein) for stars in common, arriving at a similar value for the resolving power $(R=597 \pm 72 \mathrm{~K})$. We selected stars with an rms scatter between model and observed spectra in the range 400-800 nm lower than $2 \%$, and a maximum excursion from unity in the same range within $\sim 3 \%$, derived parameters within a safe distance of the grid edges, and estimated distances under 200 pc, retaining 20 stars.
We verified that integrating over the Johnson $V$ bandpass (Cohen et al. 2003) the fluxes for the NGSL were consistent with published photometry in the Mermilliod et al. (1997) catalog, identifying a significant discrepancy ( $0.2 \mathrm{mag}$ ) for HD 193281, which we discarded after finding an even larger spread in the literature for this star $(6.3<V<6.9 \mathrm{mag})$. We also checked for variability in the Hipparcos Catalog (Perryman et al. 1997), discarding one additional star (HD 112413) with an rms scatter in $H_{p}$ larger than 0.06 mag. Only these 18 stars are discussed in the remainder of the paper.

Figure 2 shows representative fittings for 4 stars in the sample. Table 1 provides our derived atmospheric parameters for the complete sample, as well as those listed in the headers of the FITS files of the NGSL. The agreement between the effective temperatures and metallicities is fair, with our temperatures being an average of $161 \mathrm{~K}$ cooler $(\sigma=181 \mathrm{~K})$, and our metallicities an average of 0.12 dex lower $(\sigma=0.15 \mathrm{dex})$. The surface gravities show a similar offset, ours being lower by $0.18 \mathrm{dex}$, and an rms scatter $\sigma=0.16$ dex, but the discrepancies seem to be largest (by up to 0.5 dex) for the coolest and more metal rich stars in the sample.

Our conclusion about the systematics in our derived surface gravities is reinforced by comparing our results with Allende Prieto \& Lambert (1999), who compared $V$-band absolute magnitudes, computed from Hipparcos' parallaxes, and $B-V$ colors, with models of stellar interiors. Their surface gravities for 8 stars in common are an average of 0.33 larger than ours $(\sigma=0.18 \mathrm{dex})$, and show a similar trend as we found with the NGSL values, with larger discrepancies for cooler more metal-rich stars.

The comparison with metallicities from the PASTEL 

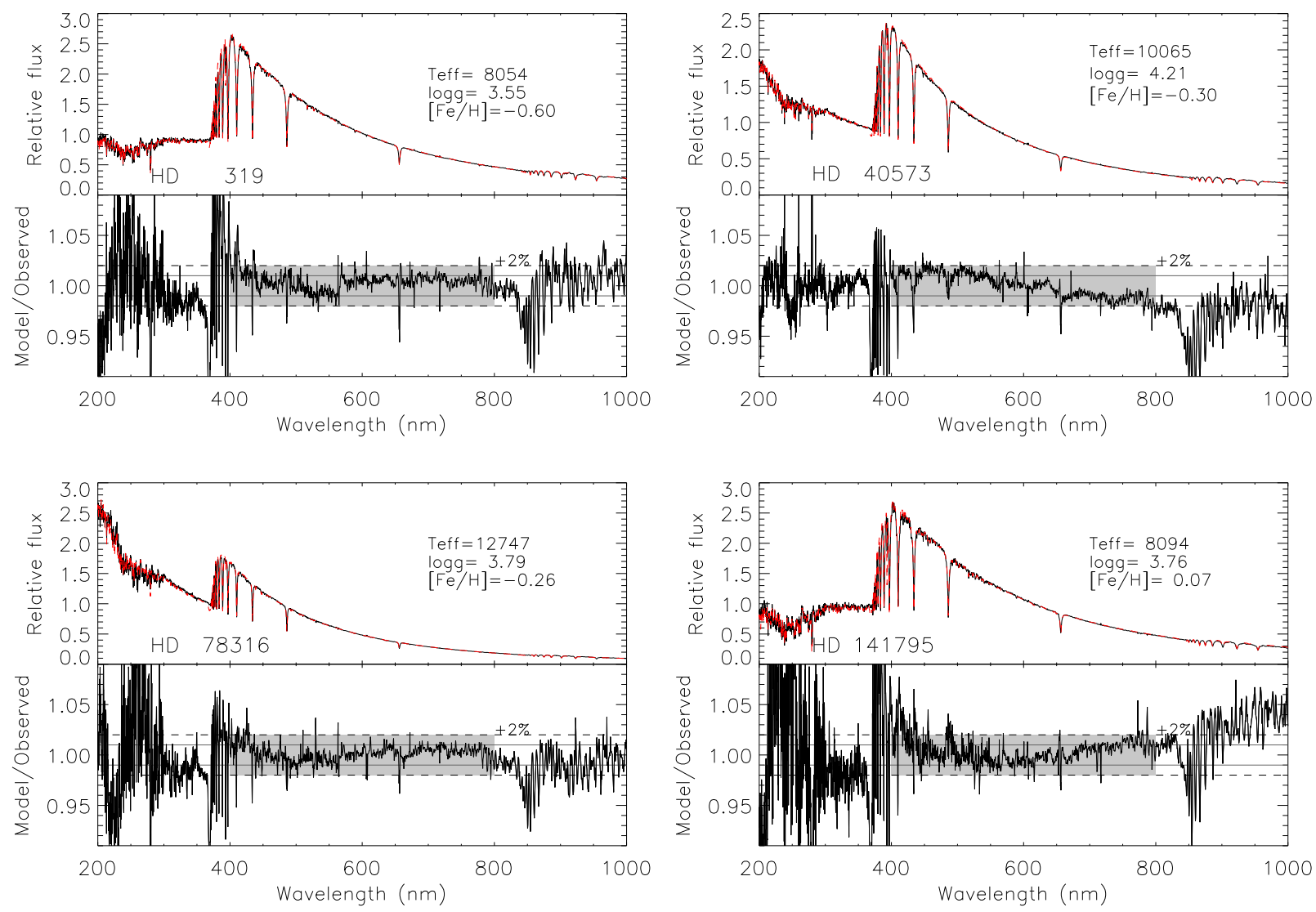

Figure 2. Upper panel: Observed (solid black line) and best-fitting model (broken red line) for four representative stars in our sample of proposed flux standards. Bottom panel: Ratio between model and observed fluxes.

bibliographical catalog (Soubiran et al. 2010), which compiles stellar atmospheric parameters determined from high resolution, high signal-to-noise spectra in the literature, shows, for eight stars in common, a mean offset of 0.02 dex, ours being lower, and significant scatter $(\sigma=0.48 \mathrm{dex})$. Our gravities are also lower than those in PASTEL by an average of $0.08 \mathrm{dex}(\sigma=0.21 \mathrm{dex})$. Lastly, our comparison with the parameters published by Koleva \& Vazdekis (2012) shows the largest spread, with standard deviations of $702 \mathrm{~K}$ in $T_{\text {eff }}, 0.5$ dex in $\log g$, and 0.6 dex in metallicity.

To evaluate the effect of neglecting reddening in our analysis, we have tested the consequences of adopting instead the reddening estimates provided by Lindler \& Heap (2008) as part of the NGSL, correcting the spectra for reddening using the mean Galactic extinction curve given by Fitzpatrick (1999), and fitting again the spectra with models. On average, the newly derived effective temperatures where $+205 K(\sigma=170 \mathrm{~K})$ warmer, the surface gravities 0.10 $\operatorname{dex}(\sigma=0.09)$ higher, and the metallicities $0.08(\sigma=0.11)$ higher than the original values. These offsets are comparable to the scatter, and consistent with our estimates of the systematic uncertainties. We therefore conclude that neglecting reddening is not a significant source of error.

We stress that even though we derive atmospheric parameters as part of our fitting process, our surface gravities and metallicities depend heavily on the Balmer and Paschen jumps and the near-UV line absorption, respectively, so they should not be taken as indicative of the true parameters of the stars, but merely as the values that provide the closest match to the NGSL spectral energy distributions. We only use them to select stars that are vetted as flux standards in the 400-800 $\mathrm{nm}$ window, and to set their reference fluxes, after scaling the models to match the observations in that window. We also use the theoretical fluxes to predict angular diameters from the comparison with the observed ones, as described in 45 , and although we find good agreement for the few stars with interferometric measurements, care must be exercised when adopting these results for the stars for which the surface gravities we derive spectroscopically and those from stellar structure models (the NGSL values in Table 1) differ. HD 18769 shows the highest difference in surface gravity, our value being lower than that from stellar structure models listed in the NGSL. Other cases for which our values are significantly smaller are HD 319, HD 38237, HD 141795, and HD 201377.

\section{ABSOLUTE FLUXES}

The absolute fluxes for the spectra in the NGSL are on the HST scale, but some caveats apply, namely E1 aperture corrections, fringing, and red light contamination to UV (G230LB) fluxes. The authors of the library have gone beyond the standard pipeline reduction to minimize the impact 
of these problems, but it is possible that the systematic errors involved are significantly larger than for other STIS observations optimized for spectrophotometric calibration (see Bohlin \& Proffitt 2015). We have computed the magnitudes in the Johnson $V$ band using the response by Cohen et al. (2003). These are provided in Table 1, together with the Johnson $V$-band magnitudes from Mermilliod et al. (1997).

Vega is the nominal zero point for the Johnson and other photometric systems, but as the best determination of the Vega spectrum evolves with time, so does the Johnson Vega magnitude and zero point - the photometry in that system remains relative to Vega, but changing Vega's magnitude is a better option than changing the magnitudes of all other stars (see the discussion by Maíz-Apellaniz 2006, 2007).

For Vega's STIS spectrum of Bohlin \& Gilliland (with the corrections described by Bohlin 2007) we find a $V=$ 0.0226 mag on the Landolt scale (using the response and inband flux provided by Cohen et al. 2003), in agreement with Bohlin's calculation $(V=0.023 \pm 0.008) \sqrt{6}$. We have similarly calculated the magnitudes of the NGSL (v2) spectra, finding good agreement between them and the $V$ magnitudes collected from Mermilliod et al. (1997): $\left\langle V-V_{\mathrm{NGSL}}\right\rangle=$ $+0.008 \pm 0.018 \mathrm{mag}^{7}$.

This comparison suggests that the zero-point of the NGSL scale is correct for our sample. Therefore, we propose to promote the stars in Tables 1 to flux standards in the $400-800 \mathrm{~nm}$ spectral window. Their best-fitting models in that spectral window are available online with this paper and from our website 8 .

\section{PREDICTED ANGULAR DIAMETERS}

The ratio between the NGSL fluxes scaled with the photometry and the computed fluxes at the surface of the star in the 400-800 $\mathrm{nm}$ window can be used to calculate the stellar angular diameters, exactly in the same way as described for Vega in 3.1 .

The results are given in Table 1 . In two cases, these results can be directly compared with angular diameters from the Palomar Testbed Interferometer (PTI) compiled by van Belle et al. (2008). These are for HD 97633 and HD 141851, for which we have derived angular diameters of $0.757 \pm 0.005$ and $0.398 \pm 0.003$ mas, respectively, while the interferometric values are $0.784 \pm 0.037$ and $0.427 \pm 0.015$ mas, respectively. Maestro et al. (2013) have recently measured the angular diameter for HD 97633 using CHARA, finding $0.740 \pm 0.024$ mas, so our value is bracketed by the PTI and CHARA measurements. Boyajian et al. (2012) has also published an angular diameter of $0.768 \pm 0.017$ mas for HD 141795, for which we find $0.756 \pm 0.007$ mas. The agreement between the two methods is good and within uncertainties, but our

\footnotetext{
6 We note that a recent photometric study by Bohlin \& Landolt (2015) shows evidence that the $V$-band response curve published by Bessell \& Murphy (2012), shifted by $-20 \AA$, gives more consistent results for about a dozen standard stars, and leads to a $V$ magnitude for Vega of 0.028 .

7 The quoted uncertainty is the standard deviation

8 http://hebe.as.utexas.edu/std/
}

estimated uncertainties are significantly smaller than those for the interferometric measurements.

There are two more objects with angular diameters in the van Belle et al. list which have not made our quality cut described in $\S 3.2$ : HD 28978 with $0.296 \pm 0.013$ mas, for which we would derive an angular diameter of $0.272 \pm 0.005$ mas, and HD 163641, with $0.178 \pm 0.027$ mas, for which we would obtain $0.174 \pm 0.006$ mas. Again, we find good consistency within error bars, but our value for HD 28978 is somewhat smaller than the interferometric diameter.

As discussed in 3.2 there are a few stars for which the surface gravities we derived spectroscopically are significantly lower from the NGSL values derived by Lindler \& Heap (2008) from the comparison with stellar evolution models. As a check, we adopted the NGSL values, refit the spectra with only $T_{\text {eff }}$ and $[\mathrm{Fe} / \mathrm{H}]$ as free parameters, and rederived angular diameters. We find that for the stars for which our gravities were up to 0.3 dex different, the inferred angular diameters differed only by less than 0.006 mas from those in Table 1.

The NGSL gravities, including for HD 141795, are in excellent agreement with the values reported by Allende Prieto \& Lambert (1999) using a similar technique, comparing absolute magnitudes from Hipparcos parallaxes with stellar models. The few discrepancies between these evolutionary gravities and those we derive from the spectrum may be associated with fast rotation or other modeling issues mentioned in the introduction.

\section{SUMMARY AND CONCLUSIONS}

We carefully examine the A-type stars with effective temperatures between 7,500 and 12,000 $\mathrm{K}$ in the Next Generation Stellar Library (Gregg et al. 2006; Heap \& Lindler 2007; Lindler \& Heap 2008) in the light of model atmospheres for appropriate atmospheric parameters. We identify eighteen stars that we propose as flux calibrators for which the models closely reproduce the observations at the $3 \%$ level in the 400-800 $\mathrm{nm}$ region. These stars have $V$ magnitudes in the range 3 to 8 , are spread in the sky $(-40<\delta<+27 \mathrm{deg})$, and can be useful when absolute flux standards are required to calibrate spectroscopic observations that demand extremely high signal-to-noise ratios and high dispersion.

The zero-point flux scale of A-type stars of the NGSL in the $V$ band appears to be consistent with the HST scale (see Bohlin 2010). Despite departures from LTE and fast rotation, classical model atmospheres can typically match the spectral energy distribution of A-type stars in the 400$800 \mathrm{~nm}$ range to better than $3 \%$. This conclusion encourages their adoption as primary flux standards for instruments targeting very bright sources, such as observations of nearby stars hosting exoplanets.

Accurate stellar radii are necessary to derive accurate planetary radii from (spectro-)photometric transits (del Burgo et al. 2010). Our study underlines the potential to derive stellar radii from spectrophotometric observations combined with accurate trigonometric parallaxes, such as those soon to be provided by Gaia. 


\section{ACKNOWLEDGEMENTS}

The authors are grateful to the referee, Ralph Bohlin, for comments that helped to improve the paper. CAP is thankful to the Spanish MINECO for support through grant AYA2014-56359-P. This work has been supported by Mexican CONACyT research grant CB-2012-183007. This research has made use of the SIMBAD database, operated at CDS, Strasbourg, France and NASA's Astrophysics Data System.

\section{REFERENCES}

Abt, H. A., Tan, H., \& Zhou, H. 1997, ApJ, 487, 365

Ali A. W., Griem H. R., 1966, PhRv, 144, 366

Allende Prieto, C. 2004, Astronomische Nachrichten, 325, 604

Allende Prieto, C., Beers, T. C., Wilhelm, R., et al. 2006, ApJ, 636, 804

Allende Prieto, C., \& Lambert, D. L. 1999, A\&A, 352, 555

Allende Prieto, C., Sivarani, T., Beers, T. C., et al. 2008, AJ, 136, 2070

Allende Prieto, C., Hubeny, I., \& Smith, J. A. 2009, MNRAS, 396, 759

Asplund, M., Grevesse, N., \& Sauval, A. J. 2005, Cosmic Abundances as Records of Stellar Evolution and Nucleosynthesis, 336, 25

Auer, L. 2003, Stellar Atmosphere Modeling, 288, 3

Aufdenberg, J. P., et al. 2006, ApJ, 645, 664

Barklem P. S., Piskunov N., O'Mara B. J., 2000, A\&AS, 142, 467

Bautista M. A., 1997, A\&AS, 122, 167

van Belle, G. T., van Belle, G., Creech-Eakman, M. J., et al. 2008, ApJS, 176, 276

Bessell, M., \& Murphy, S. 2012, PASP, 124, 140

Bohlin, R. C. 2007, The Future of Photometric, Spectrophotometric and Polarimetric Standardization, 364, 315

Bohlin, R. C. 2010, AJ, 139, 1515

Bohlin, R. C. 2014, AJ, 147, 127

Bohlin, R. C., \& Cohen, M. 2008, AJ, 136, 1171

Bohlin, R. C., \& Gilliland, R. L. 2004, AJ, 127, 3508

Bohlin, R. C., Gordon, K. D., \& Tremblay, P.-E. 2014, PASP, 126, 711

Bohlin, R. C., \& Koester, D. 2008, AJ, 135, 1092

Bohlin, R. C., \& Landolt, A. U. 2015, AJ, 149, 122

Bohlin, R., C. \& Proffitt, C. R. 2015, Instrument Science

Report, STIS 2015-01, (Baltimore:STScI)

Boyajian, T. S., McAlister, H. A., van Belle, G., et al. 2012, ApJ, 746, 101

del Burgo C., Allende Prieto C., Peacocke T., 2010, JInst, 5, 1006

Castelli, F., \& Kurucz, R. L. 2004, IAU Symp. No 210; IAU Symp. No 210, Modelling of Stellar Atmospheres, eds. N. Piskunov et al. 2003, poster A20 (arXiv:astro-ph/0405087)

Cohen, M., Wheaton, W. A., \& Megeath, S. T. 2003, AJ, 126, 1090

Cunto W., Mendoza C., Ochsenbein F., Zeippen C. J., 1993, BICDS, 42, 39

Currie, T., Plavchan, P., \& Kenyon, S. J. 2008, ApJ, 688, 597
Falcón-Barroso, J., Sánchez-Blázquez, P., Vazdekis, A., et al. 2011, A\&A, 532, A95

Fitzpatrick, E. L. 1999, PASP, 111, 63

García-Gil, A., García López, R. J., Allende Prieto, C., \& Hubeny, I. 2005, ApJ, 623, 460

Gray, R. O. 2007, The Future of Photometric, Spectrophotometric and Polarimetric Standardization, 364, 305

Gregg M. D., et al. 2006, The 2005 HST Calibration Workshop: Hubble After the Transition to Two-Gyro Mode, 209

Gulliver, A. F., Hill, G., \& Adelman, S. J. 1994, ApJl, 429, L81

Hill, G., Gulliver, A. F., \& Adelman, S. J. 2010, ApJ, 712, 250

Heap, S. R., \& Lindler, D. J. 2007, From Stars to Galaxies: Building the Pieces to Build Up the Universe, 374, 409

Hubeny, I., Hummer, D. G., \& Lanz, T. 1994, A\&A, 282, 151

Irwin A. W., 1981, ApJS, 45, 621

Koesterke, L. 2009, American Institute of Physics Conference Series, 1171, 73

Koesterke, L., Allende Prieto, C., \& Lambert, D. L. 2008, ApJ, 680, 764

Koleva, M., \& Vazdekis, A. 2012, A\&A, 538, A143

Lee, H.-W., \& Kim, H. I. 2004, MNRAS, 347, 802

Lindler, D. J., \& Heap, S. R. 2008, STIS Next Generation Spectral Library (v1), documentation at http://archive.stsci.edu/pub/hlsp/stisngsl/aaareadme.pdf van Leeuwen, Floor (2007). Hipparcos, the New Reduction of the Raw Data. Springer, Dordrecht

Maestro, V., Che, X., Huber, D., et al. 2013, MNRAS, 434, 1321

Maíz Apellániz, J. 2006, AJ, 131, 1184

Maíz Apellániz, J. 2007, The Future of Photometric, Spectrophotometric and Polarimetric Standardization, 364, 227

Mermilliod, J.-C., Mermilliod, M., \& Hauck, B. 1997, A\&AS, 124, 349

Milone, E. F., \& Sterken, C. 2011, Astrophysics and Space Science Library, 373,

Monnier, J. D., Che, X., Zhao, M., et al. 2012, ApJ, 761, L3

Nahar S. N., 1995, A\&A, 293, 967

Nelder, J. A. \& Mead, R. 1965, The Computer Journal (1965) 7, 308

Pérez Hernández, F., Claret, A., Hernández, M. M., \& Michel, E. 1999, A\&A, 346, 586

Perryman M.A.C., Lindegren L., Kovalevsky J., Hog E., et al., 1997, A\&A, 323, 49

Peterson, D. M., Hummel, C. A., Pauls, T. A., et al. 2006, Nature, 440, 896

Soubiran C., Le Campion J.-F., Cayrel de Strobel G., Caillo A., A\&A, 515, 111

Stehle C., 1994, A\&AS, 104, 509

Stehlé C., Hutcheon R., 1999, A\&AS, 140, 93

Sterken, C. 2007, The Future of Photometric, Spectrophotometric and Polarimetric Standardization, ASP Conference Series, Vol. 364, Proceedings of a conference held 8-11 May, 2006 in Blankenberge, Belgium. Edited by C. Sterken. San Francisco: Astronomical Society of the Pacific, 2007

Su, K. Y. L., et al. 2006, ApJ, 653, 675 
Tsuji T., 1964, AnTok, 9,

Tsuji, T. 1973, A\&A, 23, 411

Tsuji T., 1976, PASJ, 28, 543

van Belle, G. T.; van Belle, G.; Creech-Eakman, M. J.;

Coyne, J.; Boden, A. F.; Akeson, R. L.; Ciardi, D. R.;

Rykoski, K. M.; Thompson, R. R.; Lane, B. F., 2008; PTI

Collaboration, ApJS, 176, 276

VandenBerg, D. A., Bergbusch, P. A., \& Dowler, P. D. 2006, ApJs, 162, 375 